\title{
Efficacy of cultivated corneal epithelial stem cells for ocular surface reconstruction
}

This article was published in the following Dove Press journal:

Clinical Ophthalmology

10 September 2012

Number of times this article has been viewed

\author{
Pinnita Prabhasawat ${ }^{\prime}$ \\ Pattama Ekpo 2 \\ Mongkol Uiprasertkul ${ }^{3}$ \\ Suksri Chotikavanich' \\ Nattaporn Tesavibul' \\ 'Department of Ophthalmology, \\ ${ }^{2}$ Department of Immunology, \\ ${ }^{3}$ Department of Pathology, Faculty \\ of Medicine, Siriraj Hospital, Mahidol \\ University, Bangkok, Thailand
}

Purpose: To investigate the clinical outcomes of cultivated corneal limbal epithelial transplantation (CLET) using human amniotic membrane for corneal limbal stem-cell deficiency.

Methods: Prospective, noncomparative case series. Eighteen patients (19 eyes) with severe ocular surface diseases were chosen to undergo CLET using human amniotic membrane. Twelve eyes received auto-CLET, and seven eyes received allo-CLET. Clinical outcomes of corneal surface epithelialization, conjunctivalization, inflammation, visual acuity, graft status, and complications were observed.

Results: Corneal epithelium cultivated on amniotic membrane (two to four layers) was positive for molecular markers $p 63, A B C G 2, C K 3$, and $C K 12$. The mean patient age was $44.7 \pm 15.2$ years. A successful clinical outcome, defined as corneal epithelialization without central conjunctivalization or severe inflammation, was obtained in 14 (73.7\%) of 19 eyes (mean follow-up $26.1 \pm 13.5$ months; range 6-47). A histopathologic success, defined as absence of goblet cells at the central cornea, was achieved in $12(63.2 \%)$ eyes. Clinical failures occurred in five $(26.3 \%)$ of 19 eyes, and histopathologic failures occurred in seven (36.8\%) of 19 eyes. Survival analysis at 1 year showed that the clinical success rate was $77.9 \%$ and the pathological success rate was $72.3 \%$. Fourteen of $19(73.7 \%)$ eyes had visual acuity improvements after CLET. Six cases underwent penetrating keratoplasty; five of these grafts remained clear after $20.4 \pm 6.9$ months (range, 12-31) of follow-up. Complications included infectious keratitis (three cases) and recurrent symblepharon (one case). All complicated cases had lid abnormalities. Factors affecting the final clinical outcomes were lid abnormalities, abnormal corneal stromal beds, and complications.

Conclusion: CLET can successfully restore ocular surface damage in most cases with corneal limbal stem cell deficiency.

Keywords: limbal deficiency, limbal transplantation, corneal epithelial stem-cell transplantation, cultivated corneal epithelial stem cells

\section{Introduction}

Severe ocular surface diseases, such as Stevens-Johnson syndrome (SJS), thermal and chemical injury, and ocular cicatricial pemphigoid, can lead to severe visual loss from corneal limbal stem-cell deficiency (LSCD). ${ }^{1-4}$ These conditions can result in an abnormal corneal surface, chronic inflammation, conjunctival epithelial ingrowth (conjunctivalization) invading the cornea, corneal vascularization, poor epithelial integrity manifested as an irregular surface, recurrent epithelial erosion or persistent epithelial ulceration, and destruction of the basement membrane, leading to fibrous ingrowth. ${ }^{5}$ The clinical presentations include photophobia, corneal opacity, corneal ulcer, corneal perforation, and decreased or lost vision. Patients with LSCD are poor
Correspondence: Pinnita Prabhasawat Department of Ophthalmology, Siriraj Hospital, Mahidol University, 2 Prannok Road, Bangkoknoi District, Bangkok 10700, Thailand

Tel +6624 II 2006

Fax +6624II 1906

Email pinnita.t@gmail.com 
candidates for conventional penetrating keratoplasty (PK) due to the absence of limbal stem cells in the donor corneal button. A variety of surgical approaches, such as amniotic membrane (AM) and limbal transplantation, ${ }^{1,2,6-9}$ have been proposed to reconstruct the damaged ocular surface. In cases with total LSCD, limbal cell transplantation is needed. However, in vivo limbal transplantation has some limitations and disadvantages, such as donor size inadequacy in the autograft and a high incidence of graft rejection in allograft transplantation. Recently, cultivated corneal limbal epithelial transplantation (CLET) has been described as an interesting treatment modality for these patients. ${ }^{10-22}$ Compared to conventional keratolimbal allografts, CLET requires a smaller amount of donor tissue for cultivation, a shorter time to achieve complete corneal epithelialization, and less exposure to donor immunity. ${ }^{22}$

To the best of our knowledge, no previous report has been published in Thailand about the treatment of corneal LSCD by CLET. It is interesting to investigate the outcome and efficacy of CLET for treating severe ocular surface diseases in Thai patients. In the current study, we evaluated the use of corneal limbal epithelium cultivated on AM.

\section{Materials and methods Subjects}

The study was conducted in accordance with the principles of the Declaration of Helsinki; The Committee for the Protection of Human Participants in Research at the Faculty of Medicine, Siriraj Hospital, Mahidol University, Bangkok, Thailand (Siriraj ethics committee number 447/2550 [EC2]) approved the study. Adults with LSCD who were willing to comply with the protocol provided written informed consent before enrollment. The trial was registered with ClinicalTrials.gov, identification number NCT01237600.

The inclusion criteria selected for patients who had corneal LSCD from many causes, such as SJS, chemical or thermal burns, chronic keratitis, or multiple surgeries. LSCD diagnosis was based the absence of the limbal palisades of Vogt and the presence of conjunctival epithelial ingrowth onto the cornea (conjunctivalization), according to slit-lamp findings and subsequent confirmation by impression cytology that showed goblet cells on the corneal surface. The parameters of degree of conjunctivalization, conjunctival inflammation, conjunctival scarring, and corneal opacification were judged by the principal investigator in all cases. The conjunctival inflammation was graded as absent, mild (slight hyperemia), moderate (diffuse hyperemia), or severe (episcleral or scleral hyperemia). The conjunctival scarring was ranged from reticular subepithelial fibrosis to extensive symblepharon. Impression cytology was performed by applying nitrocellulose filter paper on the central, superior, inferior, nasal, and temporal corneal surfaces, followed by processing and staining with modified periodic acid-Schiff reagent and a modified Harris hematoxylin-eosin stain. Exclusion criteria were patients who had severe dry eye with total keratinization, patients unwilling to undergo surgery, or patients who could not comply with the follow-up schedule. For patients undergoing allografting, the exclusion criteria included patients who had contraindications or allergies to cyclosporine. Nineteen eyes of 18 patients with LSCD from chemical burns, SJS, multiple eye surgeries, drug toxicity, infectious keratitis, or idiopathic causes were recruited.

\section{Cultivation of limbal epithelial stem cells}

Under local anesthesia, superficial limbal tissue sections of $2 \times 2$ or $3 \times 1 \mathrm{~mm}$ were obtained from the contralateral normal eye (for autografting) or from a cadaveric limbal donor (for allografting), 2 to 3 weeks before the planned cultivated CLET. The limbal tissue was washed in sterile calcium and magnesium-free phosphate-buffered saline. The explants were treated with $2 \mathrm{IU} / \mathrm{mL}$ dispase $\left(\mathrm{Gibco}^{\circledR}\right.$, Life Sciences, Carlsbad, CA) at $37^{\circ} \mathrm{C}$ for 20 minutes. The denuded AM was carefully separated from its nitrocellulose membrane carrier and pulled down to adhere to the side of the bottom of the insert disc from which membrane was previously removed. The limbal epithelium was seeded onto the prepared denuded AM fixed on the culture insert disc, which was put on a sterile culture plate. The AM was obtained during elective cesarean sections with negative tests for infectious diseases and kept at $-70^{\circ} \mathrm{C}$ before use. The culture was submerged in keratinocyte growth medium for 2 to 3 weeks and then exposed to air by lowering the level of the medium (air lifting) for 1 to 2 days. The cultures were incubated at $37^{\circ} \mathrm{C}$ in a $5 \%$ carbon dioxide and 95\% air incubator, and the medium was changed on alternate days.

\section{Histologic analysis and immunohistochemical study}

Hematoxylin-eosin staining and immunohistochemical staining for stem-cell markers (transformation-related protein 63 [p63]) and differentiation markers (cytokeratin 3 [CK3], cytokeratin 12 [CK12], and cytokeratin 13 [CK13]) were performed to evaluate the epithelial characteristics of the cultivated limbal epithelium. All immunohistochemical studies were stained with the autostainer Ventana XT 
(Ventana Medical Systems, Inc, Tucson, AZ); 3,3'diaminobenzidine (Ventana Medical Systems) was used as a chromogen.

\section{Reverse-transcription polymerase chain reaction (RT-PCR)}

Gene expression in the cultivated limbal epithelium was evaluated. The RNA was quantified by its absorption at $260 \mathrm{~nm}$. Using a housekeeping gene, glyceraldehyde-3phosphate dehydrogenase (GAPDH) as an internal control, semiquantitative RT-PCR was used to analyze the mRNA expression of different molecular markers: stem-cell markers (p63 and ATP-binding cassette subfamily $\mathrm{G}$ member 2 [ABCG2]) and differentiation markers (CK3,CK12, and $C K 13)$. The resultant PCR product was analyzed by $1.5 \%$ agarose gel electrophoresis. The fidelity of the RT-PCR products was verified by comparing their sizes to the expected cDNA bands and by sequencing the PCR products.

\section{Surgical procedures}

The procedures were performed under general anesthesia. After conjunctival peritomy (360 degrees for total limbal deficiency or less for partial limbal deficiency), all abnormal fibrovascular tissue that invaded the corneal surface and symblepharon were completely removed. Mitomycin C (0.02\%; Daehan New Pharm, Seoul, South Korea) was applied to the subconjunctival space for 3 to 5 minutes, followed by vigorous washing with a balanced salt solution. The cultivated limbal epithelial sheet with an AM carrier was then removed from the culture disc, transferred onto the corneal surface, and sutured with 10-0 nylon and tissue-adhesive glue. The tissue-adhesive glue (Thai Red Cross National Blood Center, Bangkok, Thailand) was made with $1 \mathrm{~mL}$ of a solution containing 250 IU human thrombin, $2 \mathrm{mg}$ gentamycin, and $40 \mathrm{mM}$ calcium chloride and $1 \mathrm{~mL}$ of a solution containing a cryoprecipitate with $10-12 \mathrm{mg}$ fibrinogen and $12.5 \mathrm{mg}$ transamine. In cases 7 and 8 , in which the central cornea was clear, AM with a cultivated epithelial sheet was punched out centrally with a $6 \mathrm{~mm}$ trephine to avoid haze in the visual axis area. A bandage contact lens was applied at the end of the surgery.

\section{Postoperative management}

Postoperatively, preservative-free $1 \%$ methylprednisolone eye drops were prescribed hourly during week 1 , every 2 hours during week 2 , four times daily during week 3 , and then gradually tapered, depending on the degree of ocular surface inflammation. Levofloxacin eye drops $\left(\mathrm{Cravit}^{\mathbb{}}\right.$; Santen
Pharmaceutical Co, Ltd, Osaka, Japan) were used four times daily for 2 weeks. Preservative-free artificial tears and 20\% autologous serum eye drops were used frequently to promote epithelial healing. Tobramycin/dexamethasone (Tobradex ${ }^{\circledR}$; Alcon Laboratories, Inc, Fort Worth, TX) eye ointment was used at bedtime. The patients were examined daily during week 1 , at week 2 , every month for the first 6 months, and then every 2 months. Systemic cyclosporine ( 2 to $5 \mathrm{mg} / \mathrm{kg} /$ day) was prescribed after allo-CLET for 6 months and tapered gradually until it was stopped after 1 year.

\section{Secondary keratoplasty}

A secondary PK was performed $8.3 \pm 6.2$ months (range 3-16) after CLET in patients who still had a dense corneal scar involving the visual axis. The full-thickness central cornea was excised using a Hessburg-Barron trephine with a diameter of 7.5 to $8 \mathrm{~mm}$. A $0.5 \mathrm{~mm}$ oversized graft was secured by 10-0 nylon interrupted sutures or continuous sutures. Systemic cyclosporine ( 2 to $5 \mathrm{mg} / \mathrm{kg} /$ day) was prescribed postoperatively to reduce the risk of corneal graft rejection.

\section{Clinical outcome evaluation}

The postoperative visual acuity (VA) was recorded, and ocular surface manifestations were examined under a slit-lamp biomicroscope. The outcomes were evaluated according to the clinical findings (clinical outcome) and impressioncytology findings (histopathologic outcome). Clinical success was defined as stable epithelialization with or without peripheral conjunctivalization and no or mild ocular surface inflammation. Histopathologic success was defined as the absence, determined by impression cytology, of detectable goblet cells in the central cornea. Clinical failure was defined as instability of the corneal surface, such as a recurrent or persistent epithelial defect or severe inflammation with total conjunctivalization of the visual axis. Histopathologic failure was defined as the presence, determined by impression cytology, of goblet cells in the control area.

\section{Statistical analysis}

Statistical analyses were performed with SPSS 15.0 software (IBM, Armonk, NY). Kaplan-Meier analysis was performed to determine the survival of the CLETs. Factors contributing to the clinical outcomes of CLETs were determined by the Mann-Whitney test, when the factors were continuous data, and by Fisher's exact test, when the factors were in nominal scale. $P<0.05$ was considered statistically significant. 


\section{Results}

\section{Histopathologic and} immunohistochemistry results

Multiple layers of corneal epithelial cells were grown on AM from donor tissue that was $2 \mathrm{~mm} \times 2 \mathrm{~mm}$, or from $3 \mathrm{~mm} \times 1 \mathrm{~mm}$ to $2 \mathrm{~cm} \times 2 \mathrm{~cm}$, in 2 to 4 weeks. The average time of cultivation was 20.3 days (range 17-28) in the autograft group and 15.6 days (range 6-19) in the allograft group. This technique yielded two to four layers of epithelial cells seen with hematoxylin-eosin staining (Figure 1). Immunohistochemistry, studied to detect the presence of cornea-specific keratins (CK3, CK12), showed that the cultivated corneal epithelial cells were positive for CK3 and CK12. There was positive staining for CK13, which demonstrated epithelial non-keratinization. Positive staining for p63 indicated the stem-cell properties of these cultivated cells (Figure 1). RT-PCR was positive for differentiation markers $C K 3$ and $C K 12$ and for the stem-cell markers, $A B C G 2$ and p63 (Figure 2).

\section{Demographic and baseline data}

Nineteen eyes of 18 patients ( 12 men, 6 women) were enrolled in this study. The mean patient age was $44.7 \pm 15.2$ years (range 23-81). Diagnoses included chemical burn (13 eyes), allergyinduced limbal deficiency (two eyes), SJS (one eye), multiple surgeries (one eye), chronic medicamentosa (one eye), and

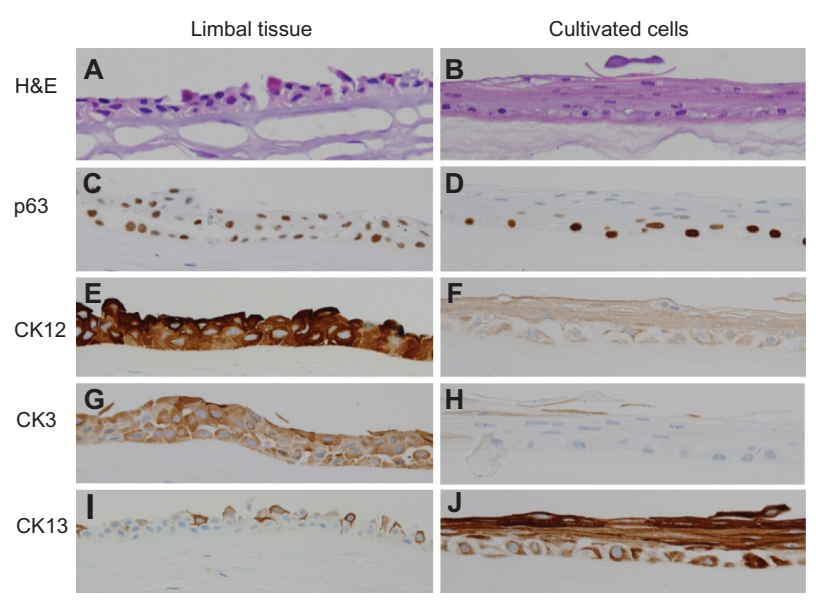

Figure I Histopathologic analysis of limbal tissue (left column) compared with cultivated corneal epithelial cells (right column). Hematoxylin-eosin staining of the limbal tissue (A) and cultivated epithelial cells (B) show a stratified epithelium consisting of two to four layers resting on an AM. Expression of p63 is positive, based on immunostaining of limbal tissue (C) and cultivated epithelial cells (D), which indicates the cellular property of the corneal epithelial stem's cultivated epithelial sheet. CK3 and CKI2 indicate the corneal epithelial phenotype is in the limbal tissue (E and $\mathbf{G}$ ) and cultivated epithelial (F and $\mathbf{H})$ cells. CKI 3 is in both limbal tissue (I) and cultivated epithelial cells $(\mathbf{J})$, indicating a nonkeratinized epithelial characteristic.

Abbreviations: AM, amniotic membrane; CK3, cytokeratin 3; CKI2, cytokeratin I2; CKI3, cytokeratin 13; H\&E, hematoxylin and eosin.

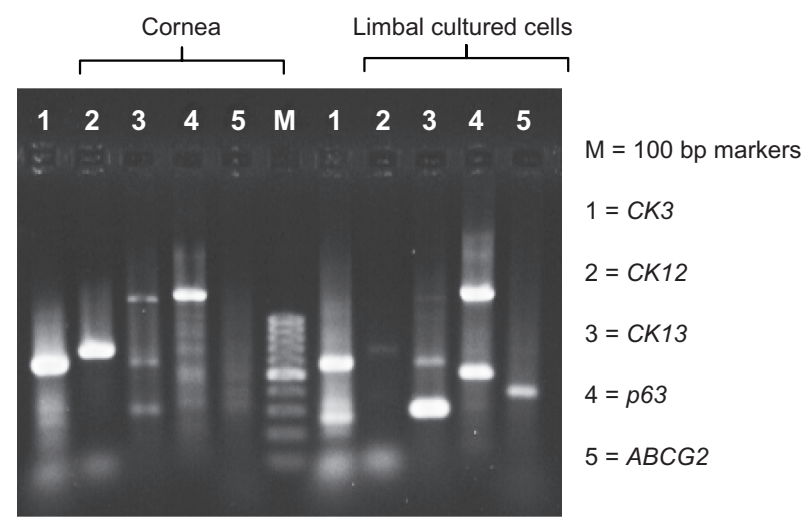

Figure 2 RT-PCR study showing expression of differentiation and stem-cell markers (CK3, CKI 2, CKI3, $A B C G 2$, and $p 63$ ).

Notes: The presence of $C K 3, C K / 2$, and $C K / 3$ indicates the nonkeratinized corneal epithelial cell property, and $A B C G 2$ and p63 indicate the stem-cell property of the limbal cultivated cells. The findings are identical to that of the immunohistochemistry study.

Abbreviations: ABCG2, ATP-binding cassette subfamily G member 2; Bp, base pair; $C K 3$, cytokeratin 3; CKI2, cytokeratin I2; CKI3, cytokeratin I3; p63, transformationrelated protein 63 ; RT-PCR, reverse-transcription polymerase chain reaction.

cytomegalovirus keratitis (one eye). Thirteen (68\%) of 19 eyes were diagnosed with total LSCD, and the other eyes were affected partially. The mean postoperative follow-up period was $26.1 \pm 13.5$ (range 6-47) months. Table 1 shows the patient demographic data.

\section{Clinical outcomes}

Clinical success was achieved in $14(73.7 \%)$ of the 19 eyes, and histopathologic success was achieved in twelve (63.2\%); clinical failure occurred in five (26.3\%), and histopathologic failure in seven (36.8\%) (Table 2). Eyes 2 and 10 achieved clinical success but failed histopathologically. Both cases had good VA, a clear central cornea, and a smooth ocular surface without vascularization or epithelial defects. However, fluorescein staining showed a localized area of conjunctival staining. The clinical success rates were lower in the autograft group $(66.7 \%, 8 / 12$ eyes) than in the allograft group (85.7\%, 6/7 eyes). However, lid abnormalities were more common in the autograft group than in the allograft group (in six eyes and in one eye, respectively). In all eyes, conjunctival inflammation decreased after CLET. Graft rejection, defined as sudden corneal epithelial damage with conjunctival inflammation, ${ }^{17}$ did not occur in any eye. The AM carrier contributed to the corneal opacification for only two months postoperatively (Figure 3B, H, and K). The AM later dissolved and disappeared without interfering with vision (Figure $3 \mathrm{~K}$ and $\mathrm{N}$ ).

The VA improved after CLET in 13 (68.4\%) of 19 eyes and remained unchanged in five eyes (cases 1, 4, 
Table I Patient characteristics and types of cultivated limbal epithelial cell transplants

\begin{tabular}{lllllll}
\hline Patient & Sex & Age (years) & Disease & Lid abnormality & $\begin{array}{l}\text { Extent of limbal } \\
\text { deficiency }\end{array}$ & $\begin{array}{l}\text { Type of cultivated } \\
\text { limbal transplant }\end{array}$ \\
\hline 1 & Female & 52 & SJS & Yes & Total & Allograft \\
2 & Female & 61 & Multiple eye surgeries & No & Total & Allograft \\
3 & Male & 80 & MMC toxicity & No & Total & Autograft \\
4 & Male & 53 & Chemical burn & Yes clock hours & Autograft \\
5 & Male & 42 & Chemical burn & Yes & Total & Autograft \\
6 & Male & 24 & Chemical burn & Yes & 8 clock hours & Autograft \\
7 & Female & 48 & CMV keratitis s/p PK & No & Total & Autograft (annular) \\
8 & Female & 44 & Chemical burn & No & Total & Autograft (annular) \\
9 & Male & 47 & Chemical burn & Yes & Total & Autograft \\
10 & Male & 49 & Chemical burn & Yes & Total & Autograft \\
11 & Male & 37 & Chemical burn & No & Allograft \\
12 & Male & 23 & Chemical burn & Yes & 4 clock hours & Autograft \\
13 & Female & 60 & Idiopathic, allergic, dry eye & No & 9 clock hours & Allograft \\
14 & Male & 35 & Chemical burn & No & Total & Autograft \\
15 & Male & 34 & Chemical burn & No & I0 clock hours & Allograft \\
16 & Male & 25 & Chemical burn & No & Allograft \\
17 & Male & 40 & Chemical burn & No & 9 clock hours & Allograft \\
18 & Female & 60 & Idiopathic, allergic, dry eye & No & No & Autograft \\
19 & Female & 26 & Chemical burn & No &
\end{tabular}

Abbreviations: SJS, Stevens-Johnson syndrome; MMC, mitomycin C; CMV, cytomegalovirus; s/P PK, status post-penetrating keratoplasty.

8, 9, 11, and 19) (Table 2). Six eyes required PK, due to dense corneal scars (Table 2; Figure 3D-F). Two eyes eventually required cataract surgery, one (case 7) after CLET (Figure 3G and I) and the other (case 11) after CLET and subsequent PK. Overall, the VA improved after CLET or CLET combined with another surgery in $14(73.7 \%)$ of 19 eyes. In case 5, however, after an uneventful keratoplasty, the patient developed infectious keratitis that developed into a central scar, resulting in finger-counting VA at 2 feet. The mean time to complete epithelialization after CLET was $11.5 \pm 18.4$ days. However, eight eyes had complete epithelialization on the first postoperative day (Table 2; Figure 3A-C and J-L). In all cases considered clinically successful, not only did the VA improve, but also the inflammation and ocular discomfort decreased postoperatively. Figure 3 shows the preoperative and postoperative clinical manifestations of representative cases in the group considered clinically successful. Severe symblepharon improved after CLET in three (75\%) of four eyes (cases 5, 6, 10, and 17).

The histopathologic sections from corneal buttons from all eyes (except case 9) that underwent subsequent PK had multiple layers of corneal epithelial cells $(16,7$, 16, 4, and 3 months after CLET in cases 2, 5, 6, 11, and 12, respectively).Those cells stained positive for $\mathrm{p} 63, \mathrm{CK} 3$, and CK12 markers. In case 9, which was considered a failure, a persistent epithelial defect developed after CLET and continued to develop after PK.
Five eyes were considered clinical failures; these were due to various causes, including symblepharon $(n=1)$, a chronic recurrent epithelial defect from lid abnormalities $(n=3)$, and infectious keratitis $(n=1)$. Two eyes (cases 2 and 10) were classified as histopathologic failures, based on the presence of goblet cells in the central corneas; but the corneas remained clear, with localized conjunctivalization staining but good VA (Table 2; Figure 4A-C).

\section{Survival analysis}

Kaplan-Meier survival analysis at 1 year showed clinical success in $77.9 \%$ of the eyes (Figure 5) and histopathologic success in $72.3 \%$ of the eyes (Figure 6). Analysis of the preoperative and postoperative factors affecting the final outcome of CLET in the allograft and autograft groups by the Mann-Whitney test (for continuous data) and Fisher's exact test (for nominal data) considered $P<0.05$ as statistically significant for all factors. Statistical analysis showed that an abnormal Schirmer's test, lid abnormality $(P=0.02)$, corneal stromal bed abnormality $(P<0.01)$, and late complications $(P=0.04)$ were found in the clinical failure group more often than in the success group. The type of surgery (autografting or allografting) did not affect the clinical outcomes.

\section{Complications}

In the current study, complications developed in five eyes during the first year. One eye had recurrent sym- 


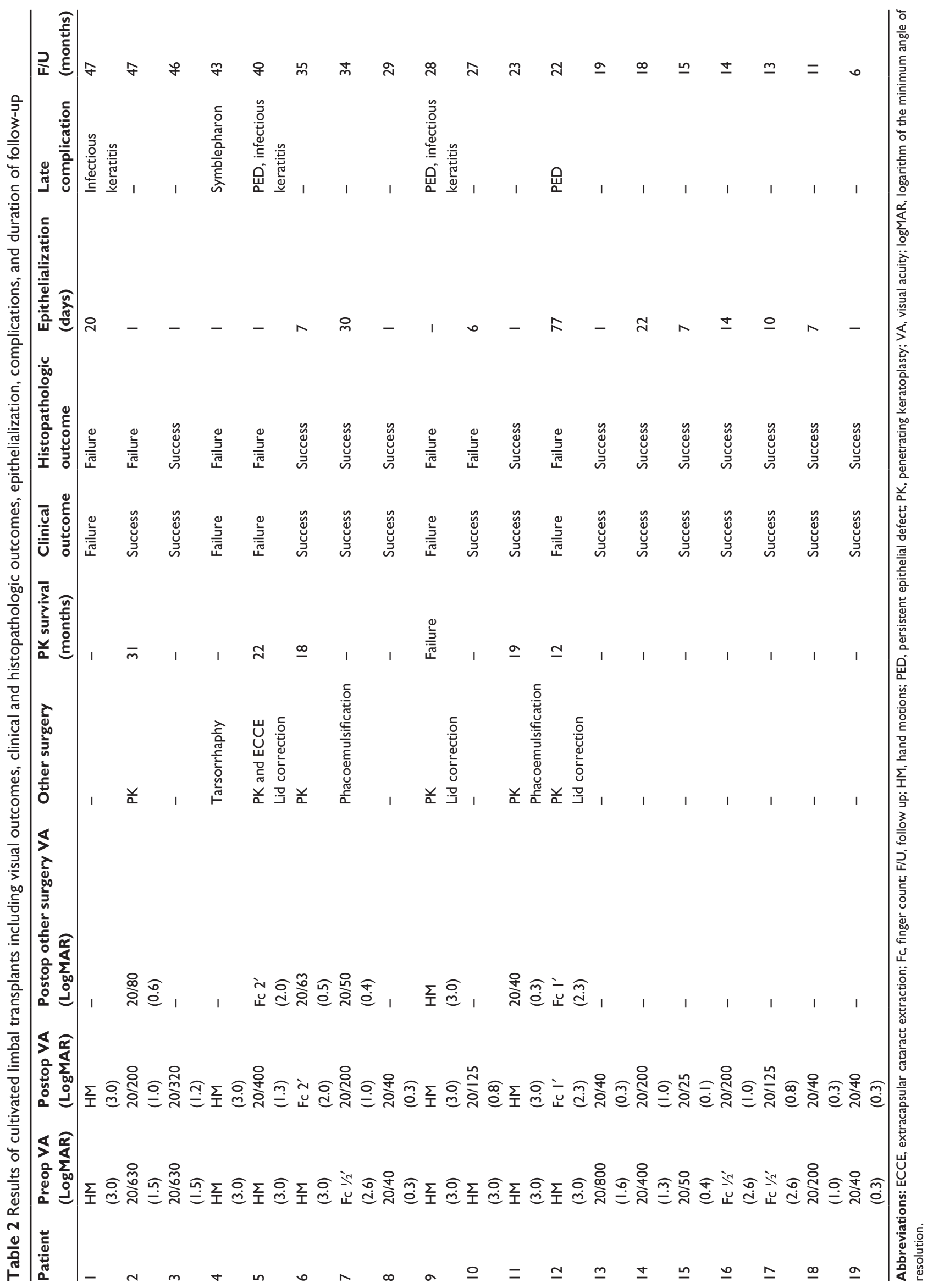



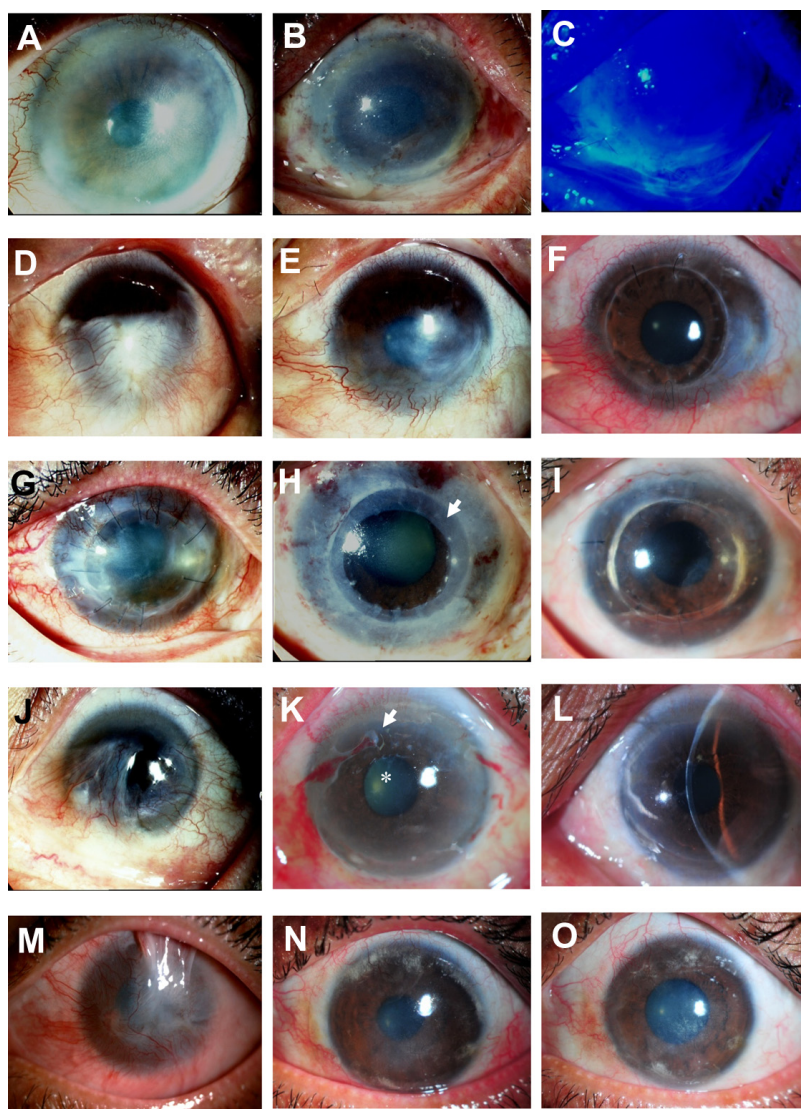

Figure 3 Representative slit-lamp photographs show a comparison of the eyes before (left column) and after (middle and right column) cultivated limbal epithelial transplantation (CLET) in the clinical success group. Patient 3 (A) undergoes auto-CLET and I day postoperatively the entire corneal surface is covered with transplanted cultivated corneal epithelium (B) confirmed by fluorescein staining, with no conjunctival cell contamination (C). Patient 6 , who has a chemical burn (D), undergoes auto-CLET, with resultant decreased corneal neovascularization postoperatively at 10 months after CLET (E). Penetrating keratoplasty is performed for optical reasons 16 months after CLET and the corneal graft remains clear 18 months after keratoplasty (F). Patient 7 underwent a previous penetrating keratoplasty to treat cytomegalovirus keratitis $(\mathbf{G})$ with severe inflammation. Two days after autoCLET with a 360-degree annular ring of CLET (arrow), the central area is spared $(\mathbf{H})$. The corneal graft has recovered and become clear with no conjunctivalization up to 34 months after CLET (I). Patient 13, with idiopathic near-total limbal stem cell deficiency bilaterally $(\mathrm{J})$, undergoes allo-CLET. The corneal epithelium becomes transparent 2 weeks postoperatively (K, arrow), dissolving amniotic membrane carrier of CLET; (star), disappeared amniotic membrane) without conjunctivalization up to 19 months (L). The visual acuity has improved from 20/800 to 20/40. Patient 16 , with total limbal stem cell deficiency with upper lid symblepharon and chronic inflammation secondary to chemical burn (M), is markedly improved after allo-CLET. The clinical appearance at $4(\mathbf{N})$ and $14(\mathbf{O})$ months postoperatively, and the visual acuity has improved to $20 / 200$ for counting fingers half a foot away.

Note: The visual acuity remains stable up to the last follow-up examination at 14 months.

blepharon, three developed infectious keratitis, and three developed a chronic recurrent epithelial defect due to a lid abnormality (Table 2). All cases with infectious keratitis also had lid abnormalities, which induced a recurrent epithelial defect. The CLET eyes that developed infectious keratitis all eventually failed treatment.
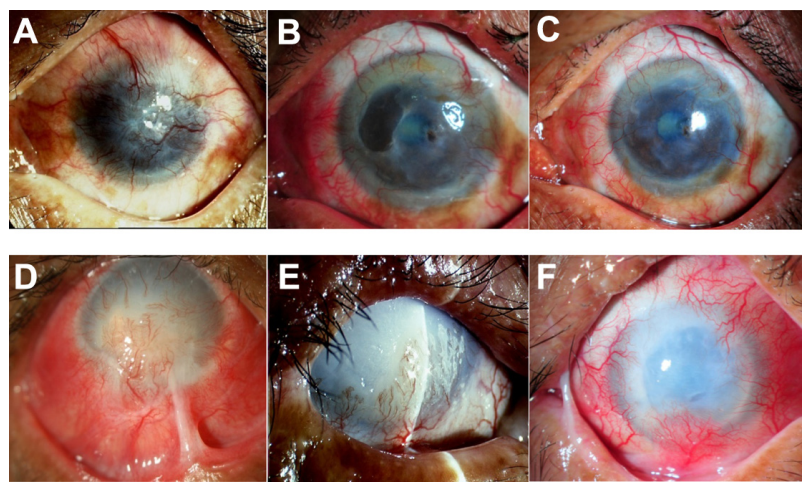

Figure 4 Slit-lamp photographs show a comparison of the eyes before (left column) and after (middle and right column) cultivated limbal epithelial transplantation (CLET) in the histopathologic failure group. Patient 10 (top row) has total limbal stem cell deficiency from a severe chemical burn (A). Postoperatively, the patient has histopathologic failure but clinical success. Cytology shows goblet cells in the central cornea at 12 months, but the central cornea has remained clear with minimal haze and no vascularization. The visual acuity at 13 (B) and 29 (C) months remains 20/I25. Patient 9 (bottom row), with limbal stem cell deficiency from a severe chemical burn, has marked lid abnormality and corneal stromal destruction (D). The patient undergoes auto-CLET. Seven months postoperatively (E), the ocular surface has less inflammation and no recurrent symblepharon. However, an epithelial defect persists and does not heal after subsequent tarsorrhaphy surgery. The patient finally undergoes penetrating keratoplasty at 9 months. Complete epithelialization has not been achieved and the clinical course was complicated by secondary infectious keratitis. The corneal graft is then rejected and eventually opaque with complete epithelialization at 30 months (F).

Note: A bandage contact lens is applied to protect against mechanical trauma from the lid and eyelashes.

\section{Discussion}

LSCD occurs after corneal limbal stem cells have been damaged permanently from burns, SJS, cicatricial pemphigoid, ectodermal dysplasia, severe vernal keratoconjunctivitis, conjunctival intraepithelial neoplasia, or multiple ocular surgeries. The affected eyes have fibrovascular invasion onto the cornea, recurrent or persistent epithelial defects, and corneal scars. Symblepharon formation has also been reported. ${ }^{2,4}$

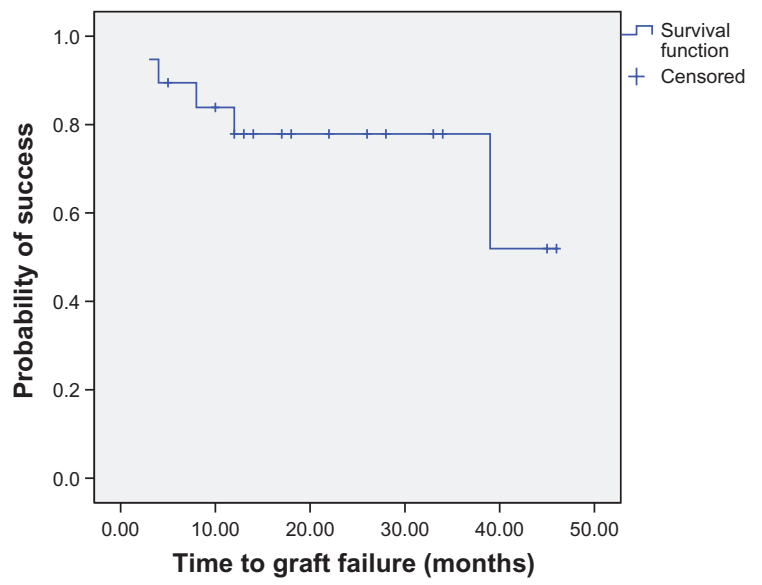

Figure 5 Survival analysis showing the clinical outcome of cultivated limbal epithelial transplantation.

Note: More than three-quarters of eyes $(77.9 \%)$ are considered clinical success I year postoperatively. 


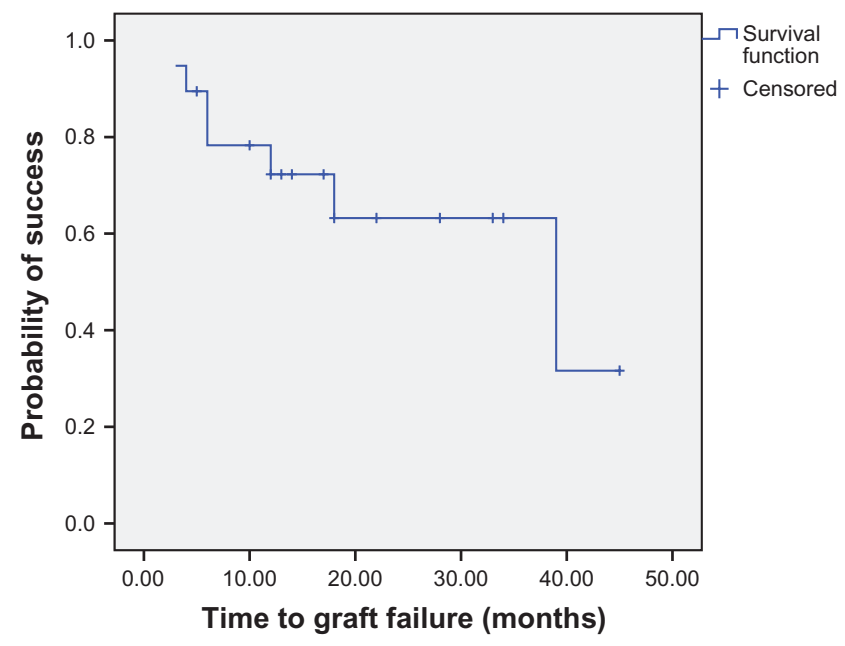

Figure 6 Survival analysis showing histopathologic outcomes of cultivated limbal epithelial transplantation.

Note: Most eyes (72.3\%) achieve histopathologic success I year postoperatively.

In some cases, chronic surface inflammation leads to ocular pain or discomfort and decreased vision. ${ }^{1,3,4}$ Previously, there was no treatment for this condition, and keratoplasty usually had a very poor outcome. Attempts have been made to find stem-cell replacements, which may help relieve the symptoms and improve the ocular surface and the vision of these patients. Thus, limbal stem-cell transplantation was first introduced to treat LSCD, ${ }^{1,2,6-9}$ followed by CLET, which has been proposed with promising results. ${ }^{10-23}$

In the current study, we cultivated corneal limbal epithelial cells using AM as a substrate without a $3 \mathrm{~T} 3$ feeder or fetal bovine serum, which differed methodologically from most previous reports ${ }^{14,15,17,24}$ but was similar to Shimazaki et $\mathrm{al}^{13}$ and Sangwan et al. ${ }^{19,25}$

The autograft cell culture needed more time than the allograft, perhaps because the superficial limbal tissue sections obtained from the contralateral normal eyes were somewhat smaller than the cadaveric limbal donors' (their smaller size reduced the sampling risk to normal eyes). The benefit of not using a 3T3 feeder is the lower chance of contamination from oncogenic cells and animal products. Moreover, the cultivated cells in the current study were positive for $p 63$ and $A B C G 2$, which indicate progenitor-cell characteristics, and stained positive for CK3 or CK12, which can be found in differentiated corneal epithelial cells. ${ }^{22}$ Nevertheless, using this technique, the cultivated corneal epithelial cells might have a rough surface, poor polarity, or fewer layers than when using the $3 \mathrm{~T} 3$ feeder technique. ${ }^{14}$ In the current study, we obtained a few layers of cultivated cells, which was similar to the results of Shimazaki et al, ${ }^{13}$ but more than the monolayer reported by Sangwan et al. ${ }^{19,25}$ The transplanted cells remained in the recipient cornea and could replicate and differentiate. This was demonstrated by the epithelial healing after corneal abrasion from lid abnormalities and the negative goblet cells in the histologically successful cases. Moreover, the immunohistochemical staining of the corneal buttons obtained from PK revealed a progenitor cells marker (p63) and corneal epithelial markers (CK3 and CK12) for as long as 16 months after CLET (cases 2 and 6).

In the current study, CLET was performed in 19 eyes with LSCD resulting from several conditions. Seven eyes received allografts and twelve eyes received autografts. This procedure improved the ocular surface condition, decreased inflammation, and reduced corneal neovascularization. Based on the current results, clinical and pathological success was achieved in $77.9 \%$ and $72.3 \%$ of the eyes, respectively, at 1 year and in $73.7 \%$ and $63.2 \%$ by the end of the study. The results were comparable to those of Rama et al, ${ }^{26}$ Pauklin et al, ${ }^{27}$ and Shimazaki et al, ${ }^{14}$ who reported success rates of $78 \%, 68 \%$, and $59 \%$, respectively. Survival analysis showed that most clinically failed cases occurred within 12 months. Interestingly, some cases that appeared clinically successful had goblet cells centrally (histopathologic failure). This finding suggests that the corneal surface could have maintained its function despite the mixed population of corneal and conjunctival epithelium. However, it might also indicate some surface conjunctival staining in the conjunctival epithelial area. ${ }^{28}$

Corneal peripheral neovascularization occurred slowly in some cases, usually during the first 3 to 6 months postoperatively, ceased to progress, and remained the same thereafter. Besides the improved corneal surface, CLET decreased conjunctival inflammation and eliminated symblepharon in all but one case. In this study, this might be due to the effect of the cultivation of the epithelial sheet on the AM, which prevents the invasion of fibrovascular tissue from the conjunctival fornix over the graft. Subconjunctival tissue treatment with $0.04 \%$ mitomycin $\mathrm{C}$ for 5 minutes has been described in some previous studies ${ }^{15,18}$ as treating residual subconjunctival fibroblasts and preventing conjunctival ingrowth following CLET. Even though a lower mitomycin C concentration $(0.02 \%)$ was used in this study, in order to reduce ocular surface toxicity, a similar favorable effect was observed.

However, the surgical results usually depended mostly on the preoperative condition of the eyes (stem-cell niche) and postoperative complications. Corneal stromal bed abnormalities, lid abnormalities, and postoperative complications such as corneal infection were significantly associated with the clinical failures. This finding confirmed the results of Shimazaki et a ${ }^{14}$ and Rama et al. ${ }^{26}$ In the present study, the clinical failure group included one case of SJS 
and severe chemical burns. All five (100\%) eyes had severe stromal bed abnormalities and severe lid abnormalities, even after lid reconstructions by an oculoplastic specialist; these abnormalities, complicated by postoperative epithelial breakdown and infection, led to the failures.

In contrast to a previous study, ${ }^{22}$ the current study found a higher clinical success rate in allograft cases $(85.7 \%$, $6 / 7$ eyes) compared with autograft cases $(66.7 \%, 8 / 12$ eyes $)$, possibly because of associated lid abnormalities, which were found in more eyes in the autograft group (six eyes) than in the allograft group (one eye).

\section{Conclusion}

In summary, the technique of cultivating corneal epithelium on AM without 3T3 and fetal bovine serum provided healthy epithelial sheets that were transplanted successfully onto of the eyes of patients with LSCD. This procedure relieved symptoms, reduced inflammation, improved the condition of the ocular surface, and increased vision. Moreover, CLET provided a better environment for a subsequent keratoplasty and perhaps a better chance of corneal graft survival. However, the success rate of this procedure depended on the extent of previous ocular damage, especially lid abnormalities and corneal stromal destruction, and on postoperative complications. This procedure is a straightforward and useful treatment for patients with LSCD, especially in cases with adequate tear flow and normal eyelids. However, for those with severe ocular surface destruction or uncorrectable lid deformities, keratoprosthesis might be a better treatment option.

\section{Acknowledgments}

This study was supported by Mahidol University. We are grateful to Assistant Professor Chulaluk Komoltri, Dr PH (Biostatistics), and Pimrapat Tengtrakulcharoen, $\mathrm{MBH}$, from the Office for Research and Development, for their assistance with statistical analysis, and to Mathuwan Srikong from the Medical Education Technology Center, Faculty of Medicine Siriraj Hospital, Mahidol University, for preparing the figures.

\section{Disclosure}

The authors have no proprietary or commercial interest in any materials or products discussed in this article.

\section{References}

1. Dua HS, Azuara-Blanco A. Limbal stem cells of the corneal epithelium. Surv Ophthalmol. 2000;44(5):415-425.

2. Dua HS, Saini JS, Azuara-Blanco A, Gupta P. Limbal stem cell deficiency: concept, aetiology, clinical presentation, diagnosis and management. Indian J Ophthalmol. 2000;48(2):83-92.

3. Prabhasawat P. Corneal limbal stem cells. Siriraj Med J. 2006;58: $728-729$.
4. Tseng SCG, Sun TT. Stem cells: ocular surface maintenance. In: Brightbill FS, editor. Corneal Surgery: Theory, Technique, and Tissue. 3rd ed. St Louis: Mosby; 1999:9-18.

5. Tseng SC. Concept and application of limbal stem cells. Eye. 1989; 3(Pt 2):141-157.

6. Holland EJ. Epithelial transplantation for the management of severe ocular surface disease. Trans Am Ophthalmol Soc. 1996;94:677-743.

7. Solomon A, Ellies P, Anderson DF, et al. Long-term outcome of keratolimbal allograft with or without penetrating keratoplasty for total limbal stem cell deficiency. Ophthalmology. 2002;109(6): $1159-1166$.

8. Tsai RJ, Tseng SC. Human allograft limbal transplantation for corneal surface reconstruction. Cornea. 1994;13(5):389-400.

9. Tsubota K, Satake Y, Kaido M, et al. Treatment of severe ocular-surface disorders with corneal epithelial stem-cell transplantation. $N$ Engl J Med. 1999;340(22):1697-1703.

10. Pellegrini G, Traverso CE, FranziAT,Zingirian M, Cancedda R, De Luca M. Long-term restoration of damaged corneal surfaces with autologous cultivated corneal epithelium. Lancet. 1997;349(9057):990-993.

11. Rama P, Bonini S, Lambiase A, et al. Autologous fibrin-cultured limbal stem cells permanently restore the corneal surface of patients with total limbal stem cell deficiency. Transplantation. 2001;72(9): $1478-1485$.

12. Tsai RJ, Li LM, Chen JK. Reconstruction of damaged corneas by transplantation of autologous limbal epithelial cells. $N$ Engl $J$ Med. 2000;343(2):86-93.

13. Shimazaki J, Aiba M, Goto E, Kato N, Shimmura S, Tsubota K. Transplantation of human limbal epithelium cultivated on amniotic membrane for the treatment of severe ocular surface disorders. Ophthalmology. 2002;109(7):1285-1290.

14. Shimazaki J, Higa K, Morito F, et al. Factors influencing outcomes in cultivated limbal epithelial transplantation for chronic cicatricial ocular surface disorders. Am J Ophthalmol. 2007;143(6):945-953.

15. Koizumi N, Inatomi T, Suzuki T, Sotozono C, Kinoshita S. Cultivated corneal epithelial transplantation for ocular surface reconstruction in acute phase of Stevens-Johnson syndrome. Arch Ophthalmol. 2001;119(2):298-300.

16. Koizumi N, Kinoshita S. Ocular surface reconstruction, amniotic membrane, and cultivated epithelial cells from the limbus. $\mathrm{Br} \mathrm{J}$ Ophthalmol. 2003;87(12):1437-1439.

17. Koizumi N, Inatomi T, Suzuki T, Sotozono C, Kinoshita S. Cultivated corneal epithelial stem cell transplantation in ocular surface disorders. Ophthalmology. 2001;108(9):1569-1574.

18. Nakamura T, Inatomi T, Sotozono C, Koizumi N, Kinoshita S. Successful primary culture and autologous transplantation of corneal limbal epithelial cells from minimal biopsy for unilateral severe ocular surface disease. Acta Ophthalmol Scand. 2004;82(4):468-471.

19. Sangwan VS, Matalia HP, Vemuganti GK, et al. Clinical outcome of autologous cultivated limbal epithelium transplantation. Indian $J$ Ophthalmol. 2006;54(1):29-34.

20. Sangwan VS, Murthy SI, Vemuganti GK, Bansal AK, Gangopadhyay N, Rao GN. Cultivated corneal epithelial transplantation for severe ocular surface disease in vernal keratoconjunctivitis. Cornea. 2005;24(4):426-430.

21. Grueterich M, Espana EM, Touhami A, Ti SE, Tseng SC. Phenotypic study of a case with successful transplantation of ex vivo expanded human limbal epithelium for unilateral total limbal stem cell deficiency. Ophthalmology. 2002;109(8):1547-1552.

22. Shortt AJ, Secker GA, Notara MD, et al. Transplantation of ex vivo cultured limbal epithelial stem cells: a review of techniques and clinical results. Surv Ophthalmol. 2007;52(5):483-502.

23. Dogru M, Tsubota K. Current concepts in ocular surface reconstruction. Semin Ophthalmol. 2005;20(2):75-93.

24. Koizumi N, Fullwood NJ, Bairaktaris G, Inatomi T, Kinoshita S, Quantock AJ. Cultivation of corneal epithelial cells on intact and denuded human amniotic membrane. Invest Ophthalmol Vis Sci. 2000; 41(9):2506-2513. 
25. Sangwan VS, Matalia HP, Vemuganti GK, et al. Early results of penetrating keratoplasty after cultivated limbal epithelium transplantation. Arch Ophthalmol. 2005;123(3):334-340.

26. Rama P, Matuska S, Paganoni G, Spinelli A, De Luca M, Pellegrini G. Limbal stem-cell therapy and long-term corneal regeneration. $N$ Engl J Med. 2010;363(2):147-155.

27. Pauklin M, Fuchsluger TA, Westekemper H, Steuhl KP, Meller D. Midterm results of cultivated autologous and allogeneic limbal epithelial transplantation in limbal stem cell deficiency. Dev Ophthalmol. 2010;45:57-70.
28. Nakamura T, Sotozono C, Bentley AJ, et al. Long-term phenotypic study after allogeneic cultivated corneal limbal epithelial transplantation for severe ocular surface diseases. Ophthalmology. 2010;117(12): 2247-2254. e1.

\section{Publish your work in this journal}

Clinical Ophthalmology is an international, peer-reviewed journal covering all subspecialties within ophthalmology. Key topics include: Optometry; Visual science; Pharmacology and drug therapy in eye diseases; Basic Sciences; Primary and Secondary eye care; Patient Safety and Quality of Care Improvements. This journal is indexed on

\section{Dovepress}

PubMed Central and CAS, and is the official journal of The Society of Clinical Ophthalmology (SCO). The manuscript management system is completely online and includes a very quick and fair peer-review system, which is all easy to use. Visit http://www.dovepress.com/ testimonials.php to read real quotes from published authors. 